\title{
QUANTIFICAÇÃO DO LINALOL NO ÓLEO ESSENCIAL DA Aniba duckei KORSTERMANS UTILIZANDO UMA NOVA COLUNA CAPILAR POLYH4-MD EM CROMATOGRAFIA GASOSA
}

Denise Domingos da Silva* e Gilberto Orivaldo Chierice

Instituto de Química de São Carlos, Universidade de São Paulo, CP 780, 13560-970 São Carlos - SP

Mário Sérgio Galhiane

Departamento de Química, Universidade Estadual Paulista, CP 473, 17033-360 Bauru - SP

Jamal da Silva Chaar

Departamento de Química, Universidade Federal do Amazonas, 3000, 69070-000 Manaus - AM

Victor Elias Mouchrek-Filho

Departamento de Tecnologia Química, Centro de Ciências Exatas e Tecnologia, Universidade Federal do Maranhão, s/n, 65950-

000 São Luís - MA

QUANTITATION OF LINALOL IN THE ESSENTIAL OIL OF Aniba duckei Korstermans USING A NEW POLYH4-MD CAPILLARY COLUMN IN GAS CHROMATOGRAPHY. In the present work a polyurethane polymer derived from castor oil was used as stationary phase for capillary gas chromatography. The polymer was obtained by reaction of hydroxylated compound and isocynate (NCO), forming urethane. Columns of $7 \mathrm{~m} \mathrm{x} \mathrm{0,25} \mathrm{mm} \mathrm{were} \mathrm{then} \mathrm{coated} \mathrm{with} \mathrm{this} \mathrm{stationary} \mathrm{phase} \mathrm{(film} \mathrm{thickness}$ of $0,25 \mu \mathrm{m}$ ) using static coating method. The Grob test was also performed. Samples of essential oil of the Aniba duckei Korstermans was then analysed in POLYH4-MD capillary column in order to evaluate its chromatographic perfomance. The linalool was found to be the major component and has been used as compound of departure for many important syntheses. Results show that the experimental columns give higher resolution and can be employed for analysis of essentials oils.

Keywords: POLYH4 - MD capillary column; linalool; polyurethane polymer.

\section{INTRODUÇÃO}

\section{Colunas capilares}

O surgimento das colunas capilares foi muito importante para a aplicação da Cromatografia Gasosa: resultou no aumento da eficiência das separações cromatográficas e, ao mesmo tempo, concorreu para o desenvolvimento de novas fases estacionárias, termicamente mais resistentes e seletivas.

As fases estacionárias podem ser constituídas por um suporte sólido, um líquido polimérico ou um suporte sólido recoberto por um líquido pouco volátil.

As colunas capilares são classificadas em WCOT ("Wall Coated Open Tubular") - onde a fase estacionária está sob a forma de filme líquido, na parede interna do tubo, SCOT ("Support Coated Open Tubular") a fase estacionária é quimicamente ligada a um suporte finamente dividido e PLOT ("Porous Layer Open Tubular") - a fase estacionária é um sólido finamente dividido ${ }^{1}$, representadas na Figura 1.

O número de fases estacionárias disponíveis para emprego das colunas capilares é reduzido quando comparado com as colunas empacotadas. Isto se deve às grandes diferenças intrínsecas entre essas colunas: na coluna empacotada a fase estacionária é depositada sobre um suporte poroso que ocupará toda a extensão da coluna, enquanto na capilar um filme da fase estacionária recobre a superfície da coluna, modificada ou não. Fases estacionárias do tipo goma que

*e-mail: dedomingos@bol.com.br - endereço atual: Departamento de Química, Universidade Federal do Rio Grande do Norte, CP 1662, 59078970 Natal - RN

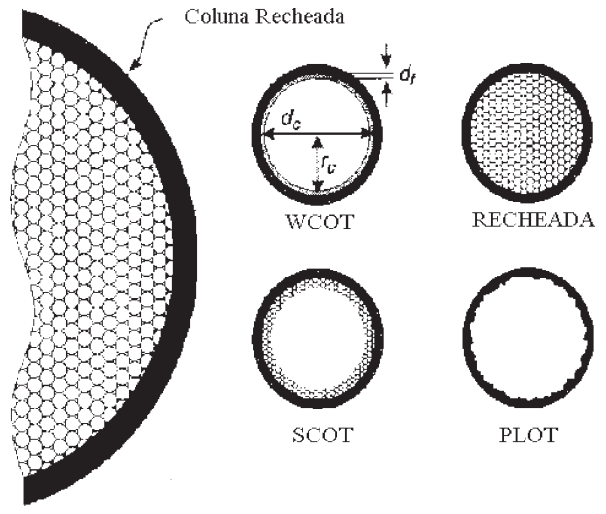

Figura 1. Tipos de colunas capilares WCOT ("Wall Coated Open Tubular" - a fase estacionária está sob a forma de um filme líquido), SCOT("Support Coated Open Tubular" - suporte recoberto com fase estacionária líquida), PLOT ("Porous Layer Open Tubular" - a fase estacionária é um sólido finamente dividido)

possuam características viscosas a alta temperatura, mantêm sua estabilidade como filmes finos e as colunas resultantes apresentam maior tempo de vida útil.

As modificações no processo de partição estão relacionadas principalmente ao tipo de fase estacionária e à característica físico-química do soluto, favorecendo diferentes tipos de interações químicas, dependendo do tipo de analito ${ }^{2}$. O desenvolvimento de novas fases poliméricas constitue objeto de grande interesse na área da Cromatografia Gasosa Capilar, devido à possibilidade de orientar a seletividade das mesmas para separações específicas, regular a faixa 
de temperatura de emprego das colunas e, assim, diversificar as aplicações da técnica.

No presente trabalho, focaliza-se a utilização de uma nova fase polimérica constituída por um polímero de poliuretano sintetizado a partir de um poliol, proveniente do óleo de mamona, na análise do linalol presente no óleo de Aniba duckei Korstermans.

\section{Polímero poliuretano}

O termo poliuretano aplica-se à classe de compostos que contêm ligação uretana, mesmo que existam outras ligações presentes. Diferentes de outros polímeros, as poliuretanas não contêm unidades uretanas repetidas de modo regular e não têm fórmula empírica que seja representativa de conjunto. Uma poliuretana típica pode conter, além dos grupos uretanos, hidrocarbonetos alifáticos e aromáticos, grupos éster, éter, uréia, amida, etc ${ }^{3}$.

$\mathrm{O}$ produto da reação química entre um grupo isocianato e um grupo hidroxila é conhecido como uretano ou uretana (1), .

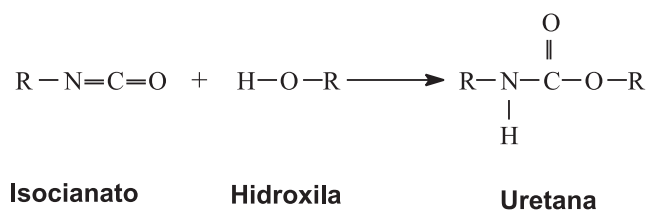

(1)

Esta reação, descoberta em 1848 por Wultz, iniciou sua aplicação comercial iniciada na década de 40, quando a Bayer desenvolveu na Alemanha, os primeiros polímeros poliuretanos ${ }^{3}$.

A polimerização dos uretanos ocorre através da reação entre um composto contendo dois ou mais grupos isocianatos em sua estrutura e um poliol. Uma grande variedade de polióis é utilizada na manufatura de poliuretanos, desde compostos naturais como o óleo de mamona, que contém grupos hidroxilas que produzem polímeros entrecruzados, até polióis sintéticos especialmente preparados para produzir polímeros com características específicas.

Os polióis são encontrados na forma de poliéteres (2) ou poliésteres (3), com diferentes funcionalidades e massa molecular.

$$
\begin{gathered}
\mathrm{CH}_{2}-\mathrm{O}-\left(\mathrm{CH}_{2}-\mathrm{CH}_{2}-\mathrm{O}\right)_{\mathrm{n}-1}-\mathrm{CH}_{2}-\mathrm{CH}_{2}-\mathrm{OH} \\
\mathrm{CH}_{2}-\mathrm{O}-\left(\mathrm{CH}_{2}-\mathrm{CH}_{2}-\mathrm{O}\right)_{\mathrm{n}-1}-\mathrm{CH}_{2}-\mathrm{CH}_{2}-\mathrm{OH} \\
\text { Poliol poliéter }
\end{gathered}
$$

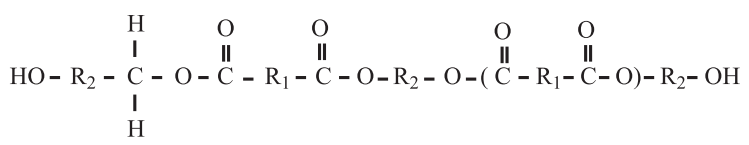

\section{Poliol poliéster}

Devido à alta reatividade dos compostos contendo grupos isocianatos, geralmente é realizada uma pré-polimerização com um poliol, aumentando sua massa molecular, deixando uma porcentagem de isocianato livre para reagir com o poliol final (4).

\section{Constituição e características do óleo essencial}

Os óleos essenciais ocupam um lugar preponderante nos merca-

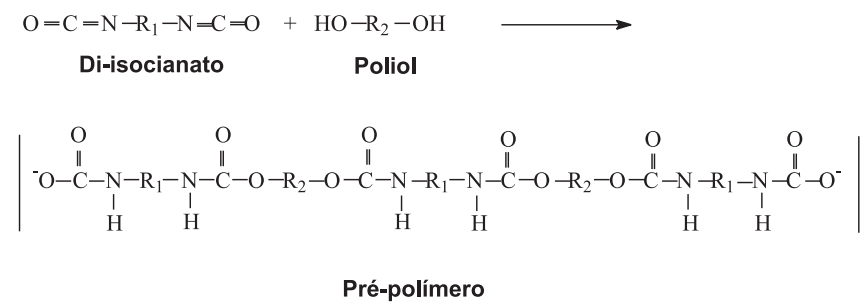

(4)

dos de farmácia, perfumaria, cosméticos e nas indústrias agro-alimentícias. São largamente usados para conferir aromas especiais em inúmeros produtos, tais como perfumes, cosméticos, sabonetes, condimentos, etc ${ }^{4,5}$.

O óleo essencial da espécie Aniba duckei Kostermans é comumente caracterizado pelo forte odor, incolor e densidade inferior à da água, sendo geralmente solúvel em solventes orgânicos usuais e em álcool $70 \%{ }^{6}$. É normalmente usado por populações locais da Amazônia para o tratamento de doenças reumáticas e de outras naturezas. Na indústria cosmética é muito utilizado, principalmente, na produção de perfumes .

O linalol (Figura 2) é o constituinte majoritário do óleo da Aniba duckei Kostermans. Outros componentes minoritários fazem parte da composição do óleo essencial, tais como o $\alpha$-terpineol, 1,8-cineol e $\alpha$ pineno. O linalol é um monoterpeno alcoólico terciário de cadeia aberta. Pode ser encontrado normalmente sob a forma de uma mistura de isômeros de posição da primeira ligação dupla. Possui um átomo de carbono assimétrico e, por isso, podem existir enantiômeros. Apresenta-se dessa forma em várias espécies ${ }^{7}$. Tem sido largamente usado como composto de partida para várias sínteses importantes, como a do acetato de linalila ${ }^{8}$, e testado como acaricida ${ }^{9}$, bactericida e fungicida ${ }^{10}$. $\mathrm{Na}$ medicina tem sido aplicado, com sucesso, como sedativo ${ }^{11,12}$ e, atualmente, estão sendo analisadas suas propriedades anticonvulsivas ${ }^{13}$. Assim, o linalol possui uma larga aplicação em várias áreas do conhecimento humano, sendo necessária sua produção em quantidades sempre crescentes. Uma característica singular do linalol no óleo é a presença do carbono assimétrico na sua estrutura, que constitui um fator determinante nas suas propriedades ${ }^{14,15}$.<smiles>C=CC(C)(O)CCC=C(C)C</smiles>

Figura 2. Estrutura do linalol

\section{PARTE EXPERIMENTAL}

\section{Instrumentos}

Todas as análises foram efetuadas no cromatógrafo a gás HP 5890 Series II, com um injetor "split-splitless" e um detector de ionização de chamas (FID). Foram usadas duas colunas capilares POLYH4-MD ( $7 \mathrm{~m} \mathrm{X} \mathrm{0,25} \mathrm{mm} \mathrm{X} \mathrm{0,25} \mathrm{\mu m)} \mathrm{e} \mathrm{outra} \mathrm{(} 25 \mathrm{~m} \mathrm{x} \mathrm{0,25} \mathrm{mm}$ $\mathrm{x} 0,25 \mu \mathrm{m}$ de espessura de filme).

\section{Síntese do polímero}

O polímero poliuretano utilizado no presente estudo foi sintetizado pelo GQATP (Grupo de Química Analítica e Tecnologia de 
Polímero) do IQSC/USP, utilizando um poliol derivado do óleo de mamona e um pré-polímero (4,4 difenilmetanodiisocianato).

\section{Colunas capilares}

Preparo das colunas capilares utilizando tubos de sílica fundida

Todas as colunas foram preparadas seguindo o processo estático $^{16}$ utilizando tubos de sílica fundida obtidos da Polimicro Technologies, Inc, Phoenix, Arizona, USA. O tubo foi previamente lavado com mistura diclorometano/pentano (1:1). Em seguida passou-se $\mathrm{N}_{2}\left(5 \mathrm{~mL} \mathrm{~min}{ }^{-1}\right)$ durante $1 \mathrm{~h}$, à temperatura ambiente para secagem e, posteriormente, preencheu-se com a solução de recobrimento contendo o polímero poliuretano.

\section{Concentração da fase}

A concentração da solução da fase estacionária foi calculada a partir da Equação $1^{16}$, estabelecendo-se o valor da espessura do filme $\left(d_{\mathrm{f}}\right)$ da fase estacionária e do diâmetro interno (d.i.) do tubo de sílica.

$d_{f}=2,5 \quad x$ d.i. $x C_{L} \%$

$\mathrm{d}_{\mathrm{f}}$ : Espessura do filme da fase estacionária $(\mu \mathrm{m})$

d.i.: Diâmetro Interno do tubo de sílica fundida $(\mu \mathrm{m})$

$\mathrm{C}_{\mathrm{L}}$ : Concentração da solução \% (massa da fase/volume da solução)

\section{Condicionamento e lavagem das colunas}

Após a etapa de impregnação da fase, as colunas foram condicionadas com programação de temperatura nas seguintes condições: $40{ }^{\circ} \mathrm{C}$ a $300{ }^{\circ} \mathrm{C}$ à $1{ }^{\circ} \mathrm{C} \mathrm{min}{ }^{-1}, 2{ }^{\circ} \mathrm{C} \mathrm{min}^{-1}, 4{ }^{\circ} \mathrm{C} \min ^{-1}, 8{ }^{\circ} \mathrm{C} \mathrm{min}{ }^{-1} . \mathrm{Em}$ seguida, procedeu-se à lavagem das colunas com $5 \mathrm{ml}$ de etanol, acetona, diclorometano, pentano e hexano.

\section{Avaliação das colunas}

A avaliação das colunas quanto à eficiência de separação e inércia (presença de cauda nos picos) foi efetuada através do teste de $\mathrm{Grob}^{16,17}$. A mistura padrão foi preparada no laboratório ${ }^{18}$ contendo os compostos constituintes do respectivo teste: hidrocarbonetos $\left(\mathrm{C}_{11}\right.$ e $\mathrm{C}_{10}$ ), 2,6-dimetilfenol, 2,4-dimetilanilina, 1-octanol, 2,3-butanediol, nonanal, ésteres $\left(\mathrm{E}_{10}, \mathrm{E}_{11}, \mathrm{E}_{12}\right)$, dicicloexilamina e ácido 2etilexanóico. As análises cromatográficas foram realizadas injetando-se $1 \mu \mathrm{L}$ da mistura em temperatura programada de $40{ }^{\circ} \mathrm{C}$ a $300{ }^{\circ} \mathrm{C}$ com taxa de aquecimento de $6{ }^{\circ} \mathrm{C} \mathrm{min}^{-1}$, split 1:100 e utilizando uma coluna capilar POLYH4-MD ( $25 \mathrm{~m}$ x 0,25 mm x 0,25 $\mu \mathrm{L}$ ). Os resultados foram comparados com os testes realizados nas colunas comerciais Carbowax-20M e HP-101 nas mesmas dimensões.

\section{Obtenção do óleo essencial}

\section{Extrator de Clevenger}

Utilizou-se um extrator de Clevenger de vidro ${ }^{19}$, acoplado a um balão de vidro de fundo redondo de $1000 \mathrm{ml}$, para a extração do óleo essencial, conforme mostrado na Figura 3. Uma manta foi usada como fonte de calor.

\section{Extração do óleo essencial}

Os óleos essenciais foram extraídos de $50 \mathrm{~g}$ de folhas secas ou galhos finos com $500 \mathrm{~mL}$ de água destilada, por hidrodestilação, em um sistema de Clevenger ${ }^{19}$ por 3,5 h, mantendo-se a temperatura em $100{ }^{\circ} \mathrm{C}$. Posteriormente os óleos foram secados em $\mathrm{Na}_{2} \mathrm{SO}_{4}$ anidro. Estas operações foram repetidas três vezes. Os óleos foram armazenados em frascos de vidro sob refrigeração, para evitar perdas de constituintes voláteis e, então, submetidos a análises.

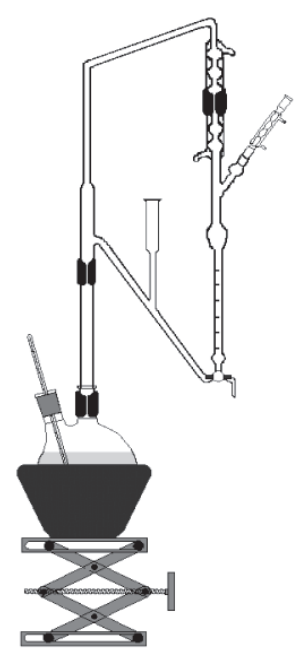

Figura 3. Extrator de Clevenger modificado

O rendimento foi calculado na relação massa/massa pela medida da densidade, observando o volume obtido no próprio sistema de extração. Para a medida da massa utilizou-se uma balança analítica da METTLER, modelo AE - 240, com precisão de $10^{-4}$ unidades.

\section{Quantificação de linalol por CG}

Foram realizadas análises por cromatografia gasosa $(\mathrm{CG})$ para quantificação do linalol pelo método do padrão externo, injetando $1 \mu \mathrm{L}$ da amostra diluída em etanol, "split" $1: 43$. Usou-se $\mathrm{H}_{2}$ como gás de arraste e velocidade linear média $\bar{\mu}=38 \mathrm{~cm} \mathrm{~s}^{-1}$, temperatura do injetor em $250{ }^{\circ} \mathrm{C}$ e do detector de ionização de chamas em $300^{\circ} \mathrm{C}$. Para a quantificação do linalol fixou-se uma programação de temperatura iniciando em $80^{\circ}$ a $7{ }^{\circ} \mathrm{C} \min ^{-1}$ até $150{ }^{\circ} \mathrm{C}$ e depois a $12{ }^{\circ} \mathrm{C} \mathrm{min}{ }^{-1}$ até $250^{\circ} \mathrm{C}(1 \mathrm{~min})$. Foi utilizada coluna Capilar POLYH4-MD $(7 \mathrm{~m}$ x $0,25 \mathrm{~mm}$ x $0,25 \mu \mathrm{m})$. As curvas analíticas foram construídas com padrões na faixa de $10^{-4}$ a $10^{-3} \mathrm{~mol} \mathrm{~L}^{-1}$. Os cálculos das concentrações foram feitos pelas equações das retas obtidas.

\section{RESULTADOS E DISCUSSÃO}

\section{Avaliação da coluna capilar POLYH4-MD}

A mistura teste de Grob foi utilizada para avaliar a seqüência de eluição dos compostos constituintes na coluna POLYH4-MD, podendo-se estabelecer comparações entre as interações químicas dos solutos com a respectiva fase e avaliar as diferenças de eluição frente às colunas comerciais de polaridade extrema (Carbowax-20M e HP101), indicado na Figura 4b e c.

A sequiência de eluição dos componentes da mistura teste na coluna POLYH4-MD indicada (Figura 4) apresenta um perfil intermediário entre as colunas de referência, HP-101 e Carbowax-20M, aproximando-se mais do perfil da primeira, com alguns deslocamentos no sentido da Carbowax-20M. Nota-se que, apesar da ordem de eluição dos hidrocarbonetos, do par 2,6 dimetilfenol/2,6-dimetilanilina e do 1-nonanal/1-octanol, nas colunas POLYH4-MD e Carbowax-20M ser a mesma, a retenção desses componentes nas referidas colunas é muito diferente.

Foram calculados os valores de número de separação para os ésteres $\mathrm{E}_{10}, \mathrm{E}_{11} \mathrm{e}_{12}{ }^{20}$. Os cálculos realizados pela Equação 2, indicam $\mathrm{SN}_{1}=16,98$ e $\mathrm{SN}_{2}=16,10$, o que confirma a similaridade entre os valores para os ésteres e reforça, também, a alta eficiência de separação da respectiva coluna capilar POLYH4-MD. Os valores encontrados para as colunas capilares CARBOWAX-20M e HP-101 

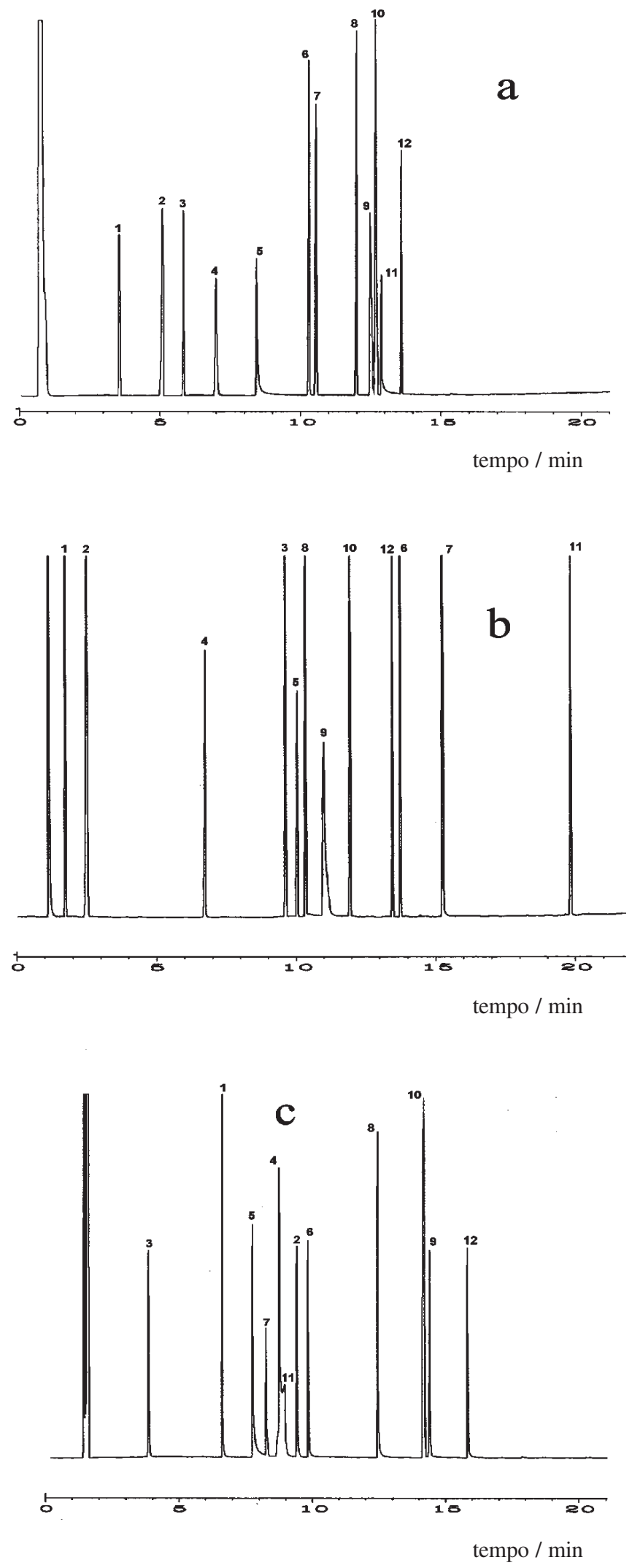

Figura 4. Avaliação do teste de GROB na (a) Coluna POLYH4-MD (25 mx

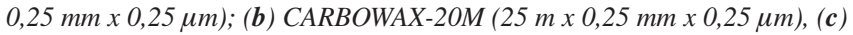
$H P-101(25 \mathrm{~m} \times 0,25 \mathrm{~mm} \times 0,25 \mu \mathrm{m})$, temperatura programada de $40{ }^{\circ} \mathrm{C}$ a $300{ }^{\circ} \mathrm{C}$ a $6{ }^{\circ} \mathrm{C} \mathrm{min}{ }^{-1}, C_{10}(1), C_{11}$ (2), 2,3-butanediol (3), 1-nonanal (4), 1 octanol (5) 2,6 - dimetilanilina (6), 2,6 -dimetilfenol (7), $E_{10}(8)$, diciclohexilamina (9), $E_{11}$ (10), Ácido 2-etilhexanóico(11) $E_{12}(12)$ foram $\mathrm{SN}_{1}=22,01, \mathrm{SN}_{2}=23,89 ; \mathrm{SN}_{1}=15,22$ e $\mathrm{SN}_{2}=15,58$, respectivamente. Os valores de número de separação apresentam-se semelhantes com diferenças somente entre $\mathrm{SN}_{1}$ e $\mathrm{SN}_{2}$. Este fator está relacionado à interação dos ésteres $\mathrm{E}_{10}, \mathrm{E}_{11}$ e $\mathrm{E}_{12}$ com as fases estacionárias, onde é correlacionado aos tempos de retenção $\left(\mathrm{t}_{\mathrm{R}}\right)$ e as larguras dos picos na meia altura.

$$
S N=\frac{t_{R(z+1)}-t_{R z}}{W_{h z}+W_{h(z+1)}}-1
$$

\section{Quantificação do linalol}

As concentrações de linalol no óleo essencial da Aniba duckei Korstermans indicadas na Figura 5 foram determinadas pela equação da reta gerada pela curva analítica y $=415,794+1,027.10^{7} \mathrm{X}$, construída pelo método do padrão externo. A curva analítica apresentou fator de correlação $\mathrm{R}=0,999$.

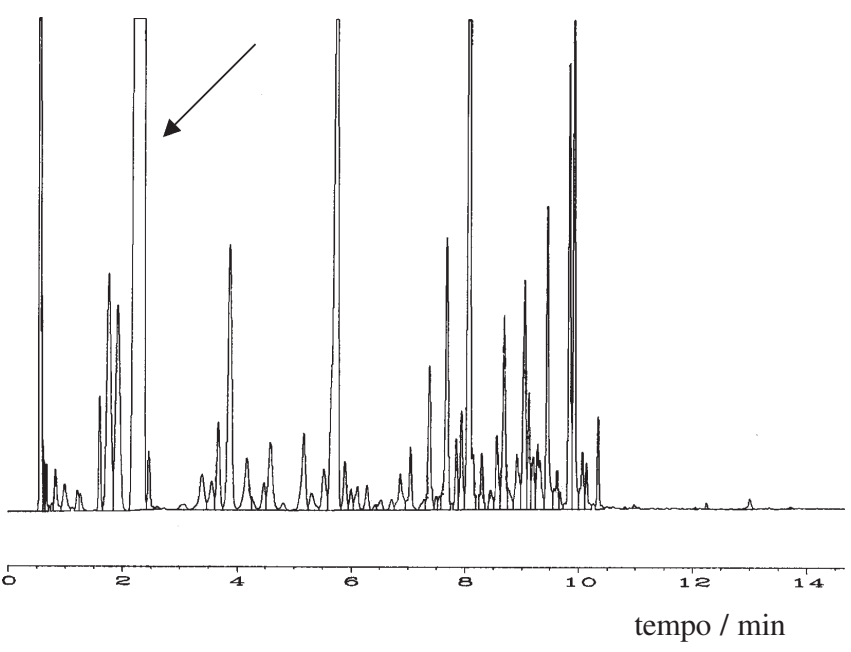

Figura 5. Cromatograma do óleo essencial das folhas da Aniba duckei Korstermans, na coluna capilar POLYH4-MD (7 m x 0,25 mm x 0,25 $\mu \mathrm{m})$, indicando o composto de referência (linalol)

Foram encontrados 55,2\% e 54, 5\% de linalol contido no óleo essencial dos galhos e das folhas da Aniba duckei Korstermans, respectivamente.

O objetivo principal desta etapa foi avaliar o desempenho da coluna capilar POLYH4-MD em relação à resposta de inércia diante de um acréscimo de concentração do linalol para a construção da curva analítica e, ao mesmo tempo, averiguar efeitos de adsorção para o respectivo soluto (linalol), quando se realiza o processo de partição.

Os percentuais obtidos para o linalol foram correspondentes aos valores encontrados por meio de quantificações realizadas com outras colunas comerciais ${ }^{21}$ (coluna capilar Carbowax-20M - $10 \mathrm{~m} \mathrm{x}$ $0,18 \mathrm{~mm} \times 0,20 \mu \mathrm{m}$ ) nas mesmas condições de análise, indicando que a coluna POLYH4-MD não apresentou problemas de adsorção com efeito de inércia satisfatório.

\section{CONCLUSÕES}

A coluna capilar POLYH4-MD apresentou alta eficiência de separação para o teste de Grob e para as amostras de óleos essenciais. Os percentuais encontrados para o linalol no óleo essencial das folhas e dos galhos da Aniba duckei Korstermans estão condizentes com a literatura ${ }^{21}$. 
Esta coluna capilar pode ser utilizada para realizar estudos relacionados com amostras complexas como óleos essenciais e também para outros tipos de compostos hidrocarbonetos ${ }^{22}$, hidrolatos ${ }^{23}$, etc, apresentando-se, devido à sua inércia, como uma coluna alternativa para os estudos qualitativos e quantitativos de óleos essenciais.

\section{AGRADECIMENTOS}

Aos órgãos de fomento CAPES e FAPESP.

\section{REFERENCIAS}

1. Hinshaw, J. V.; Ettre, L. S.; Introduction To Open Tubular Column Gas Chromatography, Advanstar Communications: USA, 1994.

2. Jennings, W.; Mittlefehldt, E.; Stremple, P.; Analytical Gas Chromatography, Academic Press: New York, 1997.

3. Saunders, J. H.; Frisch, K. C.; Polyurethane Chemistry and Technology Part I Chemistry, Interscience Publishers: New York, 1987.

4. Craveiro, A. A.; Óleos Essenciais de Plantas do Nordeste, EUFC: Fortaleza, 1981.

5. Robles, C.; Garzino, S.; Phytochemistry 1998, 50, 1343.

6. Azeredo, O. B.; Instituto de Óleos, Centro Nacional de Ensino e Pesquisas Agronômicas, Ministério da Agricultura, 1958, 15, 137.

7. Gottlieb, O. R.; Fineberg, M.; Guimarães, M. L.; Magalhães, M. T.; Maravalhas, M.; Associação Brasileira de Pesquisas sobre Plantas Aromáticas e Óleos Essenciais 1965, 11, 1 .

8. Wei, R. B.; Yuan, Z. Y.; Li, H. X.; Gazz. Chim. Ital. 1997, 127, 811.
9. Prates, H. T.; Leite, R. C.; Craveiro, A. A.; Oliveira, A. B.; J. Braz. Chem. Soc. 1998, 9, 193.

10. Belaiche, T.; Tantaoui-Elaraki, A.; Ibrahimy, A.; Sci. Alimen. 1995, 15, 571.

11. Elisabetsky, E.; Marschner J.; Souza, D. O.; Neurochem. Res. 1995, 20 461.

12. Sugawara, Y.; Hara, S.; Tamura, K.; Fujii, T.; Nakamura, K.; Masujima T.; Aoki, T.; Anal. Chim. Acta 1998, 365, 293.

13. Elisabetsky, E.; Brum, L. F. S.; Souza, D. O.; Phytomedicine 1999, 6, 107.

14. Ramos, M. C. K. V.; Aquino Neto, F. R.; Siani, A. C.; Frighetto, N.; $10^{\circ}$ Encontro Nacional de Química Analítica, Santa Maria, Brasil, 1999.

15. Casabianca, H.; Graff, J. B.; Faugier, V.; Fleig, F.; Grenier, C.; J. High Resol. Chromatogr. 1998, 21, 107.

16. Grob, K.; Making and Manipulating Capillary Columns for Gas Chromatography, Huethig : Heidelberg, 1986.

17. Lee, M. L.; Yang, F. J.; Bartle., K. D.; Open Tubular Column Gas Chromatography Theory and Practice, Jonh Wiley \& Sons Inc.: New York, 1984.

18. Grob, K.; Grob, G.; Grob Jr., K.; J. Chromatogr. 1981, 219, 13.

19. Clevenger, J. F.; American Perfumer and Essential Oil Review 1928, 467.

20. McNair, H. M.; Miller, J. M.; Basic Gas Chromatographic, Jonh Wiley \& Sons: New York, 1997.

21. Chaar, J. S.; Tese de Doutorado, Universidade de São Paulo, Brasil, 2000.

22. Domingos da Silva, D.; Galhiane, M. S.; Chieirice, G. O.; VIII Congresso Latino Americano de Cromatografia e Técnicas Afins, Buenos Aires, Argentina, 2000.

23. Moccio, C. P.; Domingos da Silva, D.; Chaar, J. S.; Galhiane, M. S.; Chierice, G. O.; $22^{\text {nd }}$ International Symposium on the Chemistry of Natural Products, São Carlos, Brasil, 2000. 